\title{
Longer screening intervals are recommended following a negative HPV test in primary cervical screening.
}

\author{
Julian Peto \& Clare Gilham
}

London School of Hygiene and Tropical Medicine

Correspondence address: London School of Hygiene \& Tropical Medicine, Keppel Street, London WC1E 7HT

Email: julian.peto@Ishtm.ac.uk

Commentary on: Dijkstra MG, van Zummeren M, Rozendaal L et al. Safety of extending screening intervals beyond five years in cervical screening programmes with testing for high risk human papillomavirus: 14 year follow-up of population based randomised cohort in the Netherlands. BMJ 2016; 355:i4924.

\section{Context}

The cervical screening programmes in the UK and the Netherlands are replacing cytology by human papillomavirus (HPV) testing. In the Netherlands women will be screened routinely at ages $30,35,40,50$ and 60 years. In the UK a decision on screening frequency has yet to be made by the National Screening Committee. Currently British women are screened with cytology every 3 years at ages 25-49 and every 5 years at ages 50-64. Pooled results of four randomised controlled trials in Europe showed that HPV testing reduces cervical cancer risk ${ }^{1}$, and the British ARTISTIC trial ${ }^{2}$ and several other studies have also shown that it gives longer protection for cervical intraepithelial neoplasia grade 3 (CIN3).

\section{Methods}

Dijkstra and colleagues describe the 14 year follow-up of the POBOSCAM trial in the Netherlands over 3 rounds of cervical screening with a 5 year interval. Almost 45,000 women were randomised in 1999-2002 between cytology alone and cytology plus HPV testing. This report compares CIN3 and cervical cancer rates in women who were HPV negative in the intervention arm and those who were cytology negative in the control arm.

\section{Findings}

The cumulative risks of CIN3 and invasive cancer were similar after 14 years in women who were HPV negative and after 9 years in women who were cytology negative. The CIN3 rate was higher in women aged 30-39, but the authors conclude that their results support the decision to extend the routine screening interval in women aged over 40 from the current 5 years with cytology to 10 years with HPV testing. They note that invasive cancer showed the opposite trend with age, and emphasise the need to keep the screening interval under review when HPV screening has been rolled out nationally.

\section{Commentary}

Among women testing negative for HPV at age 30 or over the 14 year cumulative rates for $\mathrm{CIN} 3+(\mathrm{CIN} 3$ or cancer: $0.56 \%, 95 \% \mathrm{Cl} 0.45 \%-0.70 \%)$ and for invasive cancer alone $(0.09 \%, 95 \% \mathrm{Cl} 0.04 \%-0.18 \%)$ are both extremely low. The primary aim of cervical screening is to prevent cancer, and the 22 cancers observed among HPV negative women in this very large study, suggesting an invasive cancer risk of the order of 1 in 1,000 after a 10 year screening interval, are a major addition to the evidence. Cervical cancer deaths 
are so rare in screened women that the effect on mortality of a longer screening interval can be estimated only by modelling and extrapolation. Most cancers caused by subsequent HPV infection will develop towards the end of the interval due to the lag from infection and CIN3 development to malignancy. These are likely to be diagnosed at an early stage even with a 10-year interval. Cancers present at the time of the negative HPV test would be at higher risk of being advanced or metastatic 10 years later, although modern HPV tests may already be sensitive enough to prevent most of this small but serious hazard. Collaborative pooling of data and modelling studies are needed to estimate the effects on early and advanced cancer incidence of HPV testing with screening intervals of up to 10 years at different ages, and particularly at a woman's final HPV test. CIN3+ is rare in women aged over 50 but a higher proportion are invasive cancers. The age range in POBOSCAM was 29-61 so the British protocol for women aged

below 30 will depend largely on updated results of the ARTISTIC trial, which included women from age 20.

\section{Implications for practice}

A 10-year HPV screening interval may be safe above age 40 or even 30, but the age at first HPV test and the screening interval in younger women must take into account their high HPV prevalence and CIN3 rate. In the UK the CIN3 rate at age 25-29 is 9 times higher than at 40-49. HPV infection is common among teenagers, and CIN3 detected at age 25 may have remained undiagnosed for up to 10 years.

\section{References}

1. Ronco G, Dillner J, Elfstrom KM, Tunesi S, Snijders PJ, Arbyn M, et al. Efficacy of HPV-based screening for prevention of invasive cervical cancer: follow-up of four European randomised controlled trials. Lancet. 2014 Feb 08;383(9916):524-32

2. Kitchener HC, Gilham C, Sargent A, Bailey A, Albrow R, Roberts C, et al. A comparison of HPV DNA testing and liquid based cytology over three rounds of primary cervical screening: extended follow up in the ARTISTIC trial. European journal of cancer. 2011 Apr;47(6):864-71

\section{Competing interests}

None declared. 